九州大学学術情報リポジトリ

Kyushu University Institutional Repository

\title{
OPTIMAL STOPPING PROBLEM ON FINITE MARKOV CHAIN
}

Hisano, Hiroshi

Department of Management Engineering, Tohwa University

https://doi.org/10.5109/13512

出版情報: Bulletin of informatics and cybernetics. 34 (2), pp.97-104, 2002-12. Research Association of Statistical Sciences

バージョン :

権利関係 : 


\title{
OPTIMAL STOPPING PROBLEM ON FINITE MARKOV CHAIN
}

\author{
By
}

\author{
Hiroshi Hisano*
}

\begin{abstract}
This paper studies an optimal stopping problem over a finite-horizon Markov chain on a finite-state space. First of all, we derive a recursive formula for the total number of all stopping rules in $m$-state $n$-stage stopping problem. Next we show an optimal stopping rule and give a characterization of optimal value functions.
\end{abstract}

Key Wonds and Phruses: optimal stopping, finite horizon Markov chain, total number of stopping rules

\section{Introduction}

It is wel known that a general theory of optimal stopping has been established by Snell (1952), Chow, Robbins and Siegmund (1971), Shiryaev (1978) and others. The main topic was a class of infinite-horizon problems. Recently a finite-horizon problem has been well applied to mathematical finance, in particular to fair pricing of American option, e.g. Shiryaev (1999).

On the other hand, the theory of optimal stopping is closely related to dynamic programming, e.g. Bellman (1957) and to Markov decision process, e.g. Howard (1960). A construction of optimal stopping rule for finite horizon problem is performed through backward induction. This is a basic idea of dynamic programming/Markov decision process.

In this paper we consider a class of optimal stopping problems over a finite horizon Markov chain on finite state space. We direct our attention to the total number of stopping times, optimality and characterizaion. Our approach separates the underlying process (Markov chain) and sequence of gain functions. This separation is due to the fact that the underlying process is a Markov chain. The value process over the Markov chain constitutes a minimal supermartingale.

\section{Stopping Times}

Let two integers $m \geq 1$ and $n \geq 1$ be given in this paper. We consider an $n$-stage Markov chain on state space with $m$ states. Let $\left\{X_{t}\right\}_{0}^{n}$ be a Markov chain on a finite state space $S=\left\{s_{1}, s_{2}, \ldots, s_{m}\right\}$ with a transition law $p=\{p(\cdot \cdot)\}$ :

$$
P\left(X_{t+1}=s_{j} \mid X_{t}=s_{i}\right)=p\left(s_{j} \mid s_{i}\right) .
$$

\footnotetext{
* Department of Management Engineering, TOHWA University Fukuoka 815-8510, Japan. tel +81 92--541-1152 his@tohwa-u.ac.jp
} 
We assume that the Markov chain starts at a preassigned state $s_{0} \in S ; X_{0}=s_{0}$. i times

Let $S^{i}:=\overbrace{S \times S \times \cdots \times S}$ be the direct product of $i$ state spaces $S$. We take $\Omega:=S^{n+1}$ and $N:=\{0,1, \ldots, n\}$. A mapping $\tau: \Omega \rightarrow N$ is called a stopping time if for any $t \in N$ the set $\{\tau=t\}$ is determined by random variables $\left\{X_{0}, X_{1}, \ldots, X_{t}\right\}$.The stopping time is called an $m$-state $n$-stage stopping time. Let $T:=\mathcal{T}_{0}^{n}:=\mathcal{T}_{0}^{n}(m)$ be the set of all $m$-state $n$-stage stopping times. The first question is how many stopping times there are for $m$-state $n$-stage Markov chain.

Let $f_{m}(n)$ be the the total number of $m$-state $n$-stage stopping times. Then we have the following recursive formula:

ThEOREM 2.1 .

$$
\begin{aligned}
f_{m}(n) & =1+\sum_{k=0}^{m}\left(f_{m}(n-1)-1\right)^{m-k}{ }_{m} \mathbf{C}_{k} \quad m \geq 1, n \geq 2 \\
f_{m}(1) & =2 \\
m \geq 1 . &
\end{aligned}
$$

Proof. We consider an $m$-state $n$-stage stopping time $\tau$, which does not stop immediately at starting point (on stage 0 ). Let $s_{i_{1}}, s_{i_{2}}, \cdots s_{i_{h}}$ be the states, at which $\tau$ stops on stage 1 , that is,

$$
\begin{aligned}
& \{\omega \mid \tau(\omega)=1\} \\
= & \left\{s_{0} s_{i_{1}} x_{2} \cdots x_{n}, s_{0} s_{i_{2}} x_{2} \cdots 0 x_{n}, \cdots, s s_{i_{k}} x_{2} \cdots x_{n}, x_{i} \in S, 2 \leqq i \leqq n\right\} .
\end{aligned}
$$

For each $s \in S$, we define

$$
\mu\left(x_{1} x_{2} \cdots x_{n}\right)=\tau\left(s x_{1} x_{2} \cdots x_{n}\right) .
$$

Then $\mu$ is a stopping rule of $m$-state $(n-1)$-stage stopping problem. Thus for any

$$
s \in S-\left\{s_{i_{1}}, s_{i_{2}}, \cdots, s_{i_{h}}\right\}
$$

the total number of $m$-state $n$-stage stopping rules which start at state $s$ is

$$
\left(f_{m}(n-1)-1\right)^{m-k} \text {. }
$$

Since the total number of state $x_{1} \in S$ is ${ }_{m} C_{k}$, it holds that the total number of $\tau$, which do not stop immediately at start point is

$$
\sum_{k=0}^{m}\left(f_{m}(n-1)-1\right)^{m-k}{ }_{m} \mathbf{C}_{k} .
$$

Adding one stopping time which stops immediately on stage 0 , we have the formula (2.1).

Let us take $n=1$. There exists two stopping rules: one is immidiate stop and the other is not. Thus (2.2) is valid.

\section{THEOREM 2.2.}

$$
f_{m}(n+1)=1+\left(f_{m}(n)\right)^{m} \quad n \geq 1, \quad f_{m}(1)=2 .
$$



we have

ProOF. We prove this by induction of $n$. From (2.1) and (2.2), substituting $n=2$

$$
\begin{aligned}
f_{m}(2) & =1+\sum_{k=0}^{m} 1^{m-k}{ }_{m} \mathbf{C}_{k} \\
& =1+2^{m} .
\end{aligned}
$$

Thus theorem holds for $n=1$. Now, we suppose that it hold for $n=k$. Then, by theorem 2.1, we have

$$
\begin{aligned}
f_{m}(k+2) & =1+\sum_{k=0}^{m}\left(f_{m}(k+1)-1\right)^{m-k}{ }_{m} \mathbf{C}_{k} \\
& =1+\sum_{k=0}^{m}\left(\left(f_{m}(k)\right)^{m}\right)^{m-k}{ }_{m} \mathbf{C}_{k} \\
& =1+\left(1+\left(f_{m}(k)\right)^{m}\right)^{m} \\
& =1+\left(f_{m}(k+1)\right)^{m} .
\end{aligned}
$$

This last equation shows that it holds for $n=k+1$. This completes the proof.

The recursive formula generates the following :

$$
\begin{aligned}
& f_{m}(3)=1+\left(1+2^{m}\right)^{m} \\
& f_{m}(4)=1+\left(1+\left(1+2^{m}\right)^{m}\right)^{m} \\
& f_{m}(n)=\underbrace{1+\left(1+\left(1+\left(\cdots\left(1+\left(1+2^{m}\right)^{m}\right)^{m} \cdots\right)^{m}\right.\right.}_{n=1 \text { elements }} .
\end{aligned}
$$

Table 1 shows a list of explicit numbers for two-state $(m=2)$ and three-state $(m=3)$ models.

\begin{tabular}{c|rr}
\hline$n \backslash m$ & 2 & 3 \\
\hline 0 & 1 & 1 \\
1 & 2 & 2 \\
2 & 5 & 9 \\
3 & 26 & 730 \\
4 & 677 & $389,017,001$ \\
5 & 458,330 & $5.887 \ldots \times 10^{25}$ \\
\hline
\end{tabular}

Table 1 Total numbers of $m$-stage $n$-state stopping times

\section{Optimal Stopping Problem}

Let $\left\{X_{t}\right\}_{0}^{n}$ be an $n$-stage Markov chain on $m$-state space $S=\left\{s_{1}, s_{2}, \ldots, s_{m}\right\}$ with a transition law $p=\{p(\cdot \mid \cdot)\}$ and a preassigned initial state $x_{0} \in S ; X_{0}=x_{0}$. Let $\mathcal{F}_{t}^{u}$ be the set of all subsets in $\Omega$ which are determined by random variables $\left\{X_{t}, X_{t+1}, \ldots, X_{u}\right\}$. Let us take $\mathbb{N}=\{0,1, \ldots, n\}$. A mapping $\tau: \Omega \rightarrow \mathbb{N}$ is called a stopping time if

$$
\{\tau=t\}=\left\{x_{0} x_{1} \ldots x_{n} \mid \tau\left(x_{0} x_{1} \ldots x_{n}\right)=t\right\} \in \mathcal{F}_{0}^{t} \quad \forall t \in \mathbb{N} .
$$


The stopping time $\tau$ is called $\left\{\mathcal{F}_{0}^{t}\right\}_{0}^{n}$-adapted. Let $T_{0}^{n}$ be the set of all such stopping times. Any stopping time $\tau \in \mathcal{T}_{0}^{n}$ generates a stopped state (ramdom variable) $X_{\tau}: \Omega \rightarrow$ $R^{1}$ :

$$
X_{\mathrm{r}}(\omega)=X_{\tau(\omega)}(\omega)
$$

and a stopped reward (ramdom variable) $g_{\tau}: \Omega \rightarrow R^{1}$ :

$$
g_{\tau}(\omega)=g_{\tau(\omega)}\left(X_{\tau}(\omega)\right) \text {. }
$$

We remark that the expected value $E_{x_{0}}\left[g_{\tau}\right]$ is expressed by sum of multiple sums :

$$
E_{x_{0}}\left[g_{\tau}\right]=\sum_{t=0}^{n} \sum_{\{\tau=t\}} g_{t}\left(x_{t}\right) p\left(x_{1} \mid x_{0}\right) p\left(x_{2} \mid x_{1}\right) \cdots p\left(x_{t} \mid x_{t-1}\right) .
$$

Now we consider the problem of maximizing an expected value of stopped process :

$$
\mathrm{T}_{0}\left(x_{0}\right) \quad \text { Maximize } E_{x_{0}}\left[g_{\tau}\right] \text { subject to } \tau \in \mathcal{T}_{0}^{n}
$$

\subsection{Optimality}

Let us define the sequence of functions $\left\{v_{t}\right\}_{0}^{n}$ backwardly as follows :

$$
\begin{aligned}
v_{n}\left(x_{n}\right) & =g_{n}\left(x_{n}\right) \\
v_{n-1}\left(x_{n-1}\right) & =\operatorname{Max}\left[g_{n-1}\left(x_{n-1}\right), E_{x_{n-1}}\left(v_{n}\left(X_{n}\right)\right)\right] \\
& \vdots \\
v_{1}\left(x_{1}\right) & =\operatorname{Max}\left[g_{1}\left(x_{1}\right), E_{x_{1}}\left(v_{2}\left(X_{2}\right)\right)\right] \\
v_{0}\left(x_{0}\right) & =\operatorname{Max}\left[g_{0}\left(x_{0}\right), E_{x_{0}}\left(v_{1}\left(X_{1}\right)\right)\right]
\end{aligned}
$$

where $E_{x}$ is the one-step expectation operator induced from the Markov transition matrix $p(\cdot \mid \cdot)$ :

$$
E_{x}\left(h\left(X_{t+1}\right)\right)=\sum_{y \in X} h(y) p(y \mid x)
$$

We define $\tau^{*}$ for Markov chain $\left\{X_{t}\right\}_{0}^{n}$ which starts at state $x_{0}$ on stage $0: X_{0}=x_{0}$. For $\omega=x_{0} x_{1} x_{2} x_{3} \cdots x_{n-1} x_{n}$, let

$$
\tau^{*}(\omega) \text { be the first } n \text { such that } g_{n}\left(x_{n}\right)=v_{n}\left(x_{n}\right) \text {. }
$$

Then we see that

$$
\tau^{*} \in \mathcal{T}_{0}^{n} \text {. }
$$

This is based upon the observation that

$$
\tau^{*}(\omega)=t
$$

if and only if

$$
\begin{aligned}
g_{0}\left(x_{0}\right) & <v_{0}\left(x_{0}\right) \\
g_{1}\left(x_{1}\right) & <v_{1}\left(x_{1}\right) \\
& \vdots \\
g_{t-1}\left(x_{t-1}\right) & <v_{t-1}\left(x_{t-1}\right) \\
g_{t}\left(x_{t}\right) & =v_{t}\left(x_{t}\right) .
\end{aligned}
$$


ThEOREM 3.1.

$$
E_{s_{0}}\left[g_{\tau}\right] \leqq v_{0}\left(s_{0}\right) \quad \forall \tau \in \mathcal{T}_{0}^{n}
$$

Proof. We note that

$$
\begin{aligned}
E_{s_{0}}\left[g_{\tau}\right]=\sum_{\{\tau=0\}} g_{0}\left(s_{0}\right) & +\sum_{\{\tau=1\}} p\left(x_{1} \mid s_{0}\right) g_{1}\left(x_{1}\right)+\cdots \\
& +\sum_{\{\tau=n-1\}} p\left(x_{1} \mid s_{0}\right) p\left(x_{2} \mid x_{1}\right) \cdots p\left(x_{n-1} \mid x_{n-2}\right) g_{n-1}\left(x_{n-1}\right) \\
& \quad+\sum_{\{\tau=n\}} p\left(x_{1} \mid s_{0}\right) p\left(x_{2} \mid x_{1}\right) \cdots p\left(x_{n} \mid x_{n-1}\right) g_{n}\left(x_{n}\right)
\end{aligned}
$$

From the definitions of $v_{n}$ and $v_{n-1}$, we have

the sum of the last two terms

$$
\begin{aligned}
& =\sum_{\{\tau=n-1\}} p\left(x_{1} \mid s_{0}\right) p\left(x_{2} \mid x_{1}\right) \cdots p\left(x_{n-1} \mid x_{n-2}\right) g_{n-1}\left(x_{n-1}\right) \\
& \quad+\sum_{\{\tau>n-1\}} p\left(x_{1} \mid s_{0}\right) p\left(x_{2} \mid x_{1}\right) \cdots \cdots p\left(x_{n} \mid x_{n-1}\right)\left[\sum_{x_{n} \in S} p\left(x_{n} \mid x_{n-1}\right) v_{n}\left(x_{n}\right)\right] \\
& \leqq \\
& \quad \sum_{\{\tau=n-1\}} p\left(x_{1} \mid s_{0}\right) p\left(x_{2} \mid x_{1}\right) \cdots p\left(x_{n-1} \mid x_{n-2}\right) v_{n-1}\left(x_{n-1}\right) \\
& \quad+\sum_{\{\tau>n-1\}} p\left(x_{1} \mid s_{0}\right) p\left(x_{2} \mid x_{1}\right) \cdots \cdots p\left(x_{n} \mid x_{n-1}\right) v_{n-1}\left(x_{n-1}\right) \\
& \quad \sum_{\{\tau \geq n-1\}} p\left(x_{1} \mid s_{0}\right) p\left(x_{2} \mid x_{1}\right) \cdots p\left(x_{n-1} \mid x_{n-2}\right) v_{n-1}\left(x_{n-1}\right) .
\end{aligned}
$$

Substituting this inequality into (3.2), we have

$$
\begin{aligned}
& E_{s_{0}}\left[g_{\tau}\right\} \\
\leqq & \sum_{\{\tau=0\}} g_{0}\left(s_{0}\right)+\sum_{\{\tau=1\}} p\left(x_{1} \mid s_{0}\right) g_{1}\left(x_{1}\right)+\cdots \\
& +\sum_{\{\tau=n-2\}} p\left(x_{1} \mid s_{0}\right) p\left(x_{2} \mid x_{1}\right) \cdots p\left(x_{n-2} \mid x_{n-3}\right) g_{n-2}\left(x_{n-2}\right) \\
& +\sum_{\{\tau \geq n-1\}} p\left(x_{1} \mid s_{0}\right) p\left(x_{2} \mid x_{1}\right) \cdots p\left(x_{n-1} \mid x_{n-2}\right) v_{n-1}\left(x_{n-1}\right) \\
= & \sum_{\{\tau=0\}} g_{0}\left(s_{0}\right)+\sum_{\{\tau=1\}} p\left(x_{1} \mid s_{0}\right) g_{1}\left(x_{1}\right)+\cdots \\
& +\sum_{\{\tau=n-2\}} p\left(x_{1} \mid s_{0}\right) p\left(x_{2} \mid x_{1}\right) \cdots p\left(x_{n-2} \mid x_{n-3}\right) g_{n-2}\left(x_{n-2}\right) \\
& +\sum_{\{\tau>n-2\}} p\left(x_{1} \mid s_{0}\right) p\left(x_{2} \mid x_{1}\right) \cdots p\left(x_{n-2} \mid x_{n-3}\right)\left[\sum_{x_{n-2} \in S} p\left(x_{n-1} \mid x_{n-2}\right) v_{n-1}\left(x_{n-1}\right)\right](3 .)
\end{aligned}
$$


The definition of $v_{n-2}$ shows that the sum of last two terms in (3.3) is dominated by

$$
\sum_{\{\tau \geq n-2\}} p\left(x_{1} \mid s_{0}\right) p\left(x_{2} \mid x_{1}\right) \cdots p\left(x_{n-2} \mid x_{n-3}\right) v_{n-3}\left(x_{n-2}\right) .
$$

This in turn yields

$$
\begin{aligned}
E_{s_{0}}\left[g_{\tau}\right] \leqq \sum_{\{\tau=0\}} g_{0}\left(s_{0}\right) & +\sum_{\{\tau=1\}} p\left(x_{1} \mid s_{0}\right) g_{1}\left(x_{1}\right)+\cdots \\
& +\sum_{\{\tau=n-3\}} p\left(x_{1} \mid s_{0}\right) p\left(x_{2} \mid x_{1}\right) \cdots p\left(x_{n-3} \mid x_{n-4}\right) g_{n-3}\left(x_{n-3}\right) \\
& +\sum_{\{\tau \geq n-2\}} p\left(x_{1} \mid s_{0}\right) p\left(x_{2} \mid x_{1}\right) \cdots p\left(x_{n-3} \mid x_{n-3}\right) v_{n-2}\left(x_{n-2}\right)
\end{aligned}
$$

Repeating this argument, we have

$$
E_{s_{0}}\left[g_{\tau}\right] \leqq \sum_{\{\tau=0\}} g_{0}\left(s_{0}\right)+\sum_{\{\tau \geq 1\}} p\left(x_{1} \mid s_{0}\right) v_{1}\left(x_{1}\right)
$$

Let us assume that $\{\tau=0\}=\Omega$. Then we have $v_{0}\left(s_{0}\right)=g_{0}\left(s_{0}\right)$ on $\Omega$. This implies

$$
E_{s_{0}}\left[g_{\tau}\right] \leqq v_{0}\left(s_{0}\right)
$$

Otherwise, we have $\{r=0\}=\phi$. Then we get

$$
E_{s_{0}}\left[g_{T}\right] \leqq \sum_{x_{1} \in S} p\left(x_{1} \mid s_{0}\right) v_{1}\left(x_{1}\right) \leqq v_{0}\left(s_{0}\right) .
$$

Thus we have the desired inequality (3.1).

THEOREM 3.2.

$$
E_{s_{0}}\left[g_{\tau^{*}}\right]=v_{0}\left(s_{0}\right)
$$

Proof. We note that the defintion of $\tau^{*}$ keeps the equality for all inequalities in proof of Theorem 3.1.

\subsection{Characterization}

Let two sequences of functions $\left\{f_{t}\right\}_{0}^{n},\left\{h_{t}\right\}_{0}^{n}$ on $S$ be given. Then the process $\left\{f_{t}\left(X_{t}\right)\right\}_{0}^{n}$ is said to be supermartingale (resp. martingale, stubmartingale) if $f_{t}(x) \geq$ (resp. $=$, $\leqq f_{t+1}(x) x \in X, 0 \leqq t \leqq n-1$, where

$$
T f_{t+1}(x)=E_{x}\left[f_{t+1}\left(X_{t+1}\right)\right]=\sum_{y \in X} f_{t+1}(y) p(y \mid x)
$$

denotes the expected value of tomorrow's reward function $f_{t+1}$ given today's sate $X_{t}=x$.

The process $\left\{f_{t}\left(X_{t}\right)\right\}$ is said to dominate the process $\left\{h_{t}\left(X_{t}\right)\right\}$ if $f_{t}(x) \geq h_{t}(x) \quad x \in$ , $0 \leqq t \leqq n$.

A supermartingale $\left\{f_{t}\left(X_{t}\right)\right\}$ which dominates $\left\{h_{t}\left(X_{t}\right)\right\}$ is said to be minimal if every supermartingale which dominates $\left\{h_{t}\left(X_{t}\right)\right\}$ dominates $\left\{f_{t}\left(X_{t}\right)\right\}$. 
Theorem 3.3. (Characterization) The value process $\left\{v_{t}\left(X_{t}\right)\right\}$ is the minimal supermartingale which dominates the stopping-reward process $\left\{g_{t}\left(X_{t}\right)\right\}$.

Proof. Let $\left\{u_{t}\left(X_{t}\right)\right\}$ be any supermartingale which dominates the stoppingreward process $\left\{g_{t}\left(X_{t}\right)\right\}$. Since

$$
u_{n}\left(x_{n}\right) \geq g_{n}\left(x_{n}\right)=v_{n}\left(x_{n}\right) \quad x_{n} \in S,
$$

we have

$$
u_{n-1}\left(x_{n-1}\right) \geq E_{x_{n-1}}\left(u_{n}\left(X_{n}\right)\right)=E_{x_{n-1}}\left(g_{n}\left(X_{n}\right)\right) .
$$

Further the domination impiles that

$$
u_{n-1}\left(x_{n-1}\right) \geq g_{n-1}\left(x_{n-1}\right) \quad x_{n-1} \in S .
$$

Thus we have

$$
u_{n-1}\left(x_{n-1}\right) \geq \operatorname{Max}\left[g_{n-1}\left(x_{n-1}\right), E_{x_{n-1}}\left(u_{n}\left(X_{n}\right)\right)\right]=v_{n-1}\left(x_{n-1}\right) .
$$

Repeating these arguments, we have

$$
u_{t}\left(x_{t}\right) \geq v_{t}\left(x_{t}\right) \quad 0 \leqq t \leqq n, x_{t} \in S
$$

This shows that $\left\{v_{t}\left(X_{t}\right)\right\}$ is minimal, which completes the proof.

Let $\mathfrak{M}$ be the set of all sequences of functions $\left\{u_{t}\right\}_{0}^{n}$ such that $\left\{u_{t}\left(X_{t}\right)\right\}$ is a supermartingale which dominates the stopping-reward process $\left\{g_{t}\left(X_{t}\right)\right\}$.

THEOREM 3.4. If there exists $\left\{u_{t}\right\}_{0}^{n} \in \mathfrak{M}$ such that any $\left\{h_{t}\right\}_{0}^{n} \in \mathfrak{M}$ enjoys the property that $\left\{h_{t}\left(X_{t}\right)\right\}$ dominates $\left\{u_{t}\left(X_{t}\right)\right\}$. Then it holds that

$$
u_{t}\left(x_{t}\right)=v_{t}\left(x_{t}\right) \quad 0 \leqq t \leqq n, \forall x_{t} \in S .
$$

Proof. Since $\left\{u_{t}\right\}_{0}^{n} \in \mathfrak{M}$, we have from Theorem 3.3

$$
u_{t}\left(x_{t}\right) \geq v_{t}\left(x_{t}\right) \quad 0 \leqq t \leqq n, \forall x_{t} \in S .
$$

On the other hand, $\left\{v_{t}\right\}_{0}^{n} \in \mathfrak{M}$ enjoys the domination property. This implies that the reverse inequality. Thus we have the equality, which completes the proof.

\section{Acknowledgement}

Author would like to thank Prof. Seiichi Iwamoto for helpful comments and suggestions. 


\section{References}

Bellman, R.E. (1957). Dynamic Programming, NJ:Princeton Univ. Press.

Chow, Y.S., Robbins, H. and Siegmund, D. (1971). Great Expectations: The Theory of Optimal Stopping, Boston: Hunghton Mifflin Company.

Howard, R.A. (1960). Dynamic Programming and Markov Processes, Mass.: MIT Press.

Iwamoto, S. (2002). Optimal stopping in fuzzy envinonment, Proc. 9-th Bellman Continuum, Beijing, 264-269.

Shiryaev, A.N. (1978). Optimal Stopping Rules, New York: Springer-Verlag.

Shiryaev, A.N. (1999). Essentials of Stochastic Finance, Singapore: World Scientific.

Snell, J.L. (1952). Applications of martingale system theorems, Transactions of the American Mathematical Society,73, 171-176. 